Pure Mathematical Sciences, Vol. 3, 2014, no. 2, 79 - 85

HIKARI Ltd, www.m-hikari.com

http://dx.doi.org/10.12988/pms.2014.424

\title{
Homothetic Exponential Motions with Generalized Quaternions
}

\author{
Faik Babadağ \\ Kırıkkale University, Art \& Science Faculty \\ Kırıkkale, Turkey
}

Copyright (c) 2014 Faik Babadağ. This is an open access article distributed under the Creative Commons Attribution License, which permits unrestricted use, distribution, and reproduction in any medium, provided the original work is properly cited.

\begin{abstract}
In this paper, we give the real matrix representations of generalized quaternions, and then, the concept of homothetic exponential motions is discussed and their velocities, accelerations obtained, Also, the paper gives some formula and facts about exponential motions which are not generally known for generalized quaternions.
\end{abstract}

Mathematics Subject Classification: 15A90, 53A05, 53A17

Keywords: Homothetic exponential motion, Generalized Quaternions, Matrix Equation, Diagonal Matrix, Similarity

\section{Introduction}

To state the geometry of the motion of a point in the motion of space is significant in the study of kinematices or spatial mechanisms or in physics. The geometry of such a motionof a point or a line has a number of applications in geometric modeling and model-based manufacturing of mechanical products or in the design of robotic motions. Hacisalihoğlu [1], [2] showed some properties of 1-parameter homothetic motion in Euclidean space $\mathrm{E}^{n}$. Subsequently, Kula and Yaylı [3], and [4] expressed Hamilton motions bymeans of Hamilton operators in semi-Euclidean spaces $\mathrm{E}_{2}^{4}$ and showed that this motions, are a homothetic motion.In this paper, first, we give $4 \mathrm{x} 4$ matrices representations of the generalized quaternions and of their algebraic properties. Furthermore, we 
define that a motions by using these matrices, and show that this motion is a homothetic exponential motions. We show that the homothetic exponential motions has only one pole point at every instant $t$, and define that this motion has only one acceleration center of high order at every instant t. We hope that these results will contribute to the study of space kinematics and physics.

\section{Generalized Quaternions}

A generalized quaternion is a number of form

$$
\omega=\omega_{0}+i \omega_{1}+j \omega_{2}+k \omega_{3}
$$

where $\omega_{0}, \omega_{1}, \omega_{2}, \omega_{3}$ are reel numbers called the components of $\omega$, and $i, j, k$ are arbitrary "units" which satisfy the relations

$$
\left\{\begin{array}{c}
i^{2}=-\alpha, j^{2}=-\beta, k^{2}=-\alpha \beta, i j=-j i=k \\
j k=\beta i=-k j, k i=\alpha j=-i k ; \quad \alpha, \beta \in R .
\end{array}\right\}
$$

The set of all generalized quaternions are denoted by $H_{\alpha_{\beta}}$ and

$$
H_{\alpha_{\beta}}=\left\{\omega \mid \omega=\omega_{0}+\omega_{1} i+\omega_{2} j+\omega_{3} k ; \omega_{0-3}, \alpha, \beta \in R\right\} .
$$

Write $\omega_{0}+\omega_{1} i+\omega_{2} j+\omega_{3} k$ as $\left(\omega_{0}+\omega_{1} i\right)+j\left(\omega_{2}+\omega_{3} i\right)=z_{1}+j z_{2}$ where $z_{1}, z_{2}$ are complex numbers" $C$ "

Furthermore, rules of multiplication and addition of two complex numbers have the following properties [7].

$$
\left\{\begin{array}{c}
\left(z_{1}+j z_{2}\right) \cdot\left(z_{3}+j z_{4}\right)=\left(z_{1} z_{3}-z_{2} z_{4}\right)+j\left(z_{1} z_{4}+z_{2} z_{3}\right), \\
\left(z_{1}+j z_{2}\right)+\left(z_{3}+j z_{4}\right)=\left(z_{1}+z_{3}\right)+j\left(z_{2}+z_{4}\right), \\
z_{1}+j z_{2}=z_{3}+j z_{4} \Longleftrightarrow z_{1}=z_{3} \text { and } z_{2}=z_{4} .
\end{array}\right\}
$$

In particular, in eq.[4], if we put $z_{1}=a$ and $z_{2}=i b$ with $a, b \in C$. In that case, $H_{\alpha_{\beta}}$ is a lagebra and non -associative. This algebra is called generalized quaternion algebra and are denoted by $H_{\alpha_{\beta}}$, Note that one of the basis of this algebra is $\{1, i, j, k\}$ and the dimension is 4 . It is clear, if $\alpha=\beta=1$ then $H_{\alpha_{\beta}}$ real quaternion, and if $\alpha=1, \beta=-1$ then $H_{\alpha_{\beta}}$ split quaternion.

Definition 2.1 (The addition and multiplication ): Addition of two generalized quaternions $u, w \in H_{\alpha_{\beta}}$ is defined by

$$
u+w=\left(u_{0}+w_{0}\right)+\left(u_{1}+w_{1}\right) i+\left(u_{2}+w_{2}\right) j+\left(u_{3}+w_{3}\right) k,
$$

whereas mutiplication is defined by

$$
\left\{\begin{array}{c}
u . w=\left(u_{0} w_{0}-\alpha u_{1} w_{1}-\beta u_{2} w_{2}-\alpha \beta u_{3} w_{3}\right)+ \\
\left(u_{0} w_{1}+u_{1} w_{0}+\beta u_{2} w_{3}-\beta u_{3} w_{2}\right) i+ \\
\left(u_{0} w_{2}+u_{2} w_{0}+\alpha u_{3} w_{1}-\alpha u_{1} w_{3}\right) j+ \\
\left(u_{0} w_{3}+u_{1} w_{2}-u_{2} w_{1}+u_{3} w_{0}\right) k .
\end{array}\right\}
$$


Definition 2.2 (The Concept of Conjugate for Generalized Quaternions):The conjugate of $\omega$ is defined to be

$$
\bar{\omega}=\omega_{0}-\omega_{1} i-\omega_{2} j-\omega_{3} k
$$

Definition 2.3 (Norms of Generalized Quaternions) :The norm of $\omega$ is defined to be

$$
\begin{aligned}
& |\omega|=\sqrt{\omega \bar{\omega}}=\sqrt{\omega_{0}^{2}+\alpha \omega_{1}^{2}+\beta \omega_{2}^{2}+\alpha \beta \omega_{3}^{2}}, \\
& N_{\omega}=\omega_{0}^{2}+\alpha \omega_{1}^{2}+\beta \omega_{2}^{2}+\alpha \beta \omega_{3}^{2} .
\end{aligned}
$$

Although $H_{\alpha_{\beta}}$ is nonassociative, it is still an alternative, flexible and division algebra over $\mathbf{R}$, that is, for all $u, w \in H_{\alpha_{\beta}}$, the following equalities hold:

1. $u(u \cdot \omega)=u^{2} \cdot \omega$

2. $(u \omega) \cdot \omega=u \cdot \omega^{2}$

3. $u(u \cdot \omega)=(u \omega) \cdot \omega$

4. $|u . \omega|=|u||\omega|$

5. $\omega^{-1}=\frac{\bar{\omega}}{|\omega|^{2}}$

For the generalized quaternion algebra $H_{\alpha_{\beta}}$. It is well known that through the bijective map [5], [6]

$\phi: \omega=\omega_{0}+\omega_{1} i+\omega_{2} j+\omega_{3} k \in H_{\alpha_{\beta}} \longrightarrow \phi(\omega)=\left[\begin{array}{cccc}\omega_{0} & -\alpha \omega_{1} & -\beta \omega_{2} & -\alpha \beta \omega_{3} \\ \omega_{1} & \omega_{0} & -\beta \omega_{3} & \beta \omega_{2} \\ \omega_{2} & \alpha \omega_{3} & \omega_{0} & -\alpha \omega_{1} \\ \omega_{3} & -\omega_{2} & \omega_{1} & \omega_{0}\end{array}\right]$,

$H_{\alpha_{\beta}}$ is algebraically isomorphic to the matrix algebra

$$
M=\left\{\left[\begin{array}{cccc}
\omega_{0} & -\alpha \omega_{1} & -\beta \omega_{2} & -\alpha \beta \omega_{3} \\
\omega_{1} & \omega_{0} & -\beta \omega_{3} & \beta \omega_{2} \\
\omega_{2} & \alpha \omega_{3} & \omega_{0} & -\alpha \omega_{1} \\
\omega_{3} & -\omega_{2} & \omega_{1} & \omega_{0}
\end{array}\right]: \omega_{0-3}, \alpha, \beta \in R\right\}
$$

and $\phi(\omega)$ is a faithful real matrix representation of $\omega$.

Lemma 2.4 Let $u, \omega \in H_{\alpha_{\beta}}, k \in R,[6]$.

1. $u=\omega \Leftrightarrow \phi(u)=\phi(\omega)$ 
2. $\phi(u+\omega)=\phi(u)+\phi(\omega)$

3. $\phi(u \cdot \omega)=\phi(u) \cdot \phi(\omega)$

4. $\phi(k . \omega)=k . \phi(\omega) ; \quad \phi(1)=I_{4}$

5. $\phi(\bar{\omega})=\phi^{T}(\omega)$

Lemma 2.5 Let $\omega=\omega_{0}+\omega_{1} i+\omega_{2} j+\omega_{3} k \in H_{\alpha_{\beta}}$, and denote $\omega=$ $\left[\omega_{0}, \omega_{1}, \omega_{2}, \omega_{3}\right]^{T}$, called the vector representation of $\omega$. Then for all $u, \omega \in H_{\alpha_{\beta}}$, we have

$$
u \omega=\phi(u) \omega
$$

Proof 2.6 Let $\omega=\phi(\omega) \eta_{4}^{T}, \eta_{4}=[1,0,0,0]$. We find by eq.[7] that

$$
u \omega=\phi(u \omega) \eta_{4}^{T}=\phi(u) . \phi(\omega) \eta_{4}^{T}=\phi(u) \omega .
$$

Definition 2.7 A matrix $X$ is called a quasi-orthogonal matrix if $X^{T} \varepsilon X=\varepsilon$ and $\operatorname{det} X=1$, here $\varepsilon=\left[\begin{array}{cccc}1 & 0 & 0 & 0 \\ 0 & \alpha & 0 & 0 \\ 0 & 0 & \beta & 0 \\ 0 & 0 & 0 & \alpha \beta\end{array}\right]$ and $\alpha, \beta \in R$.

\section{Homothetic Exponential Motions With Gen- eralized Quaternions}

Definition 3.1 Let $f(t)=e^{t A}$ and $B(t)=h(t) f(t)$ be the orthogonal matrix. Where $h(t) \neq$ constant, $t \in R$.

$$
\begin{aligned}
& {\left[\begin{array}{c}
X \\
1
\end{array}\right]=\left[\begin{array}{cc}
B & C \\
0 & 1
\end{array}\right] \cdot\left[\begin{array}{c}
X_{0} \\
1
\end{array}\right]} \\
& {\left[\begin{array}{c}
X \\
1
\end{array}\right]=\left[\begin{array}{cc}
h A & C \\
0 & 1
\end{array}\right] \cdot\left[\begin{array}{c}
X_{0} \\
1
\end{array}\right]}
\end{aligned}
$$

which is called homothetic exponential motion. The homothetic scale $h$ the elements of $f$ are continuously differentiable function of a real parameters $t$.

Where $X$ and $X_{0}$ correspond to position vectors the same point with respect to the rectangularcoordinate systems of the moving space $R_{0}$ and the fixed space $R$, At first time $t=t_{0}$ we consider coordinate systems of $R_{0}$ and $R$ coincident. Then homothetic exponential motions of $R_{0}$ with respect to $R$ will be denoted by $R_{0} \backslash R$. $\forall \mathrm{t}$, 
Let $f^{\prime}=A f$, and suppose $C \neq 0$ then

$$
B^{\prime}=\frac{d B}{d t}=h^{\prime} f+h f^{\prime}=\left(h^{\prime}+h A\right) f .
$$

where $h=h(t)$ is a scalar matrix, its inverse and transpose are

$$
h^{-1}=\frac{1}{h} \cdot I, h^{T}=h
$$

respectively. Since $f$ is an orthogonal matrix, the inverse of $B$ is

$$
B^{-1}=h^{-1} f^{T}, f^{-1}=f^{T}
$$

Theorem 3.2 The matrix defined by the equation in eq.[20] is a quasiorthogonal matrix.

Proof 3.3 Since $A^{T} \varepsilon A=\varepsilon$ and $\operatorname{det} A=1$.

Theorem 3.4 The homothetic exponential motion given by eq.[16] is regular for all $n$ and it is independent of $h$.

Proof 3.5 $B^{\prime}=h^{\prime} f+h f^{\prime}=\left(h^{\prime}+h A\right) f=h f(A+\lambda I)$, where if we define $\lambda(t)=$ $-\frac{h^{\prime}(t)}{h(t)}$, then last equation is

$$
B^{\prime}=h f(A-\lambda I)
$$

from eq. [21] we find that

$$
\operatorname{det} B^{\prime}=\operatorname{det}(h f) \operatorname{det}(A-\lambda I) \text {. }
$$

$A s \operatorname{det} B^{\prime}=0$, that is, $B^{\prime}$ is singular, we get

$$
h=0 \text { or } \operatorname{det}(A-\lambda I)=0 .
$$

Here $h \neq 0$. Otherwise, the motion will be pure translation. $B^{\prime}$ is always regular.

\section{POLE POINTS OF EXPONENTIAL MO- TIONS}

Definition 4.1 To find the pole points, we have to solve the equation

$$
B^{\prime} X_{0}+C^{\prime}
$$


Any solution of the equation in eq.[30] is a pole point of the motion at that instantin $R_{0}$. The equation in eq.[30] has only one solution,

$$
X_{0}=-\left(B^{\prime}\right)^{-1} C
$$

at every $t$-instant [7].

Theorem $4.2 f^{\prime}(t)$ is a derivation of $f(t)$.then, the pole points corresponding to each $t$ - instant in $R_{0}$ is the rotation by $\left(B^{\prime}\right)^{-1}$ of the speed vector $C^{\prime}$ of the translation vector at the momoent.

Proof 4.3 Since $B^{\prime}$ is quasi-orthogonal [4], then the matrix $\left(B^{\prime}\right)^{-1}$ is orthogonal. Thus it makes a rotation.

Theorem 4.4 If $f$ is a orthogonal nxn matrix, the $n^{\text {th }}$-order derivatives of $B$ are given by

$$
B^{(n)}=\left[\sum_{j=o}^{n}\left(\begin{array}{c}
n \\
j
\end{array}\right) h^{(n-j)} A^{j}\right] f .
$$

Proof 4.5 The proof of this theoram can be defined by induction. For $n=1$,

$$
B^{\prime}=\left(h^{\prime}+h A\right) f=\left[\sum_{j=0}^{1}\left(\begin{array}{l}
1 \\
j
\end{array}\right) h^{(1-j)} A^{j}\right] f .
$$

Thus we have shown that it is true for (n-1) and

$$
B^{(n-1)}=\left[\sum_{j=o}^{n-1}\left(\begin{array}{c}
n-1 \\
j
\end{array}\right) h^{(n-j-1)} A^{j}\right] f .
$$

It can now be shown that it is true for $n$. Thus, for $n$ :

$$
B^{(n)}=\left[B^{(n-1)}\right]^{\prime}=\left[\sum_{j=o}^{n-1}\left(\begin{array}{c}
n-1 \\
j
\end{array}\right) h^{(n-j-1)} A^{j}\right] f .
$$

By using the formulas the sresult can be given as follows:

$$
B^{(n)}=\left[\sum_{j=o}^{n}\left(\begin{array}{c}
n \\
j
\end{array}\right) h^{(n-j)} A^{j}\right] f .
$$

which proves the theorem.

Theorem 4.6 In spaces of n-dimension the high order velocities of homothetic exponential motions are given by 
Proof 4.7 The proof of this theorem can be defined by induction. For $n=1$,

$$
X^{\prime}=B^{\prime} X_{0}+B X_{0}^{\prime}+C^{\prime} \text { or } X^{\prime}=\left[\sum_{j=o}^{1}\left(\begin{array}{l}
1 \\
j
\end{array}\right) B^{(1-j)} X_{0}^{j}+C^{\prime}\right] .
$$

Shown that it is true for $n=1$ and $(n-1)$. For $n$ :

$$
X^{(n)}=n 0 B^{(n)} X_{0}+n 1 B^{(n-1)} X_{0}^{\prime}+\ldots+n n B X_{0}^{(j)}+C^{(n)}
$$

or

$$
X^{(n)}=\sum_{j=o}^{n}\left(\begin{array}{c}
n \\
j
\end{array}\right) B^{(n)} X_{0}^{(j)}+C^{(n)}
$$

and we find

$$
X^{(n)}=\sum_{j=o}^{n}\left[\sum_{i=o}^{n-j}\left(\begin{array}{c}
n-j \\
i
\end{array}\right) h^{(n-j-i)} A^{i}\right] f X_{0}^{(j)}+C^{(n)} .
$$

\section{References}

[1] H.H.Hacisalihoğlu, On The Rolling of one curve or surface upon another. Mathematical Proceeding of the R. Irish Acad, vol. 71(2),13-17.(1971).

[2] H.H.Hacısalihoğlu, Motions and Quaternions theory. Gazi Üniversity, Ankara.(1983).

[3] L. Kula, Y. Yayli, Homothetic Motions in semi - Euclidean spaces $E_{2}^{4}$. Mathematical Proceeding of the R. Irish Academy, Vol. 105, section A, Num.1,9-15.(2005).

[4] O.P. Agrawal, Hamilton Operators and Dual Quaternions in Spatial Kinematics, Mech-Mach Theory (22), 569-575.(1987).

[5] Y. Yayli, Homothetic Motions at Mech. Mach Theory 27(3), 303305.(1992).

[6] L.Feng, Decomposition of Some Type of Quaternions Matrices. Linear and Multilinear Algebra.Volume 58, Issue 4.(2010).

[7] A.P. Aydın, Homothetic exponential motions and their velocities. The journal of Firat University, 2(2),33-39.(1987).

\section{Received: February 5, 2014}

